Gut and Liver, Vol. 11, No. 1, January 2017, pp. 112-120

\title{
Integrative Analysis of Microarray Data to Reveal Regulation Patterns in the Pathogenesis of Hepatocellular Carcinoma
}

\author{
Juan Chen ${ }^{1}$, Zhenwen Qian ${ }^{2}$, Fengling $\mathrm{Li}^{3}$, Jinzhi Li ${ }^{4}$, and $\mathrm{Yi} \mathrm{Lu}^{5}$ \\ ${ }^{1}$ Four Ward, Taian Disabled Soldiers Hospital of Shandong Province, Taian, ${ }^{2}$ Department of Inspection, Affiliated Hospital of Jining Medical \\ College of Shandong Province, Jining, ${ }^{3}$ Nursing Department, Taian Disabled Soldiers Hospital of Shandong Province, Taian, Departments of \\ ${ }^{4}$ Pathology and ${ }^{5}$ Orthopedics, People's Hospital of Zhangqiu City, Zhangqiu, China
}

\begin{abstract}
Background/Aims: The integration of multiple profiling data and the construction of a transcriptional regulatory network may provide additional insights into the molecular mechanisms of hepatocellular carcinoma (HCC). The present study was conducted to investigate the deregulation of genes and the transcriptional regulatory network in HCC. Methods: An integrated analysis of HCC gene expression datasets was performed in Gene Expression Omnibus. Functional annotation of the differentially expression genes (DEGs) was conducted. Furthermore, transcription factors (TFs) were identified, and a global transcriptional regulatory network was constructed. Results: An integrated analysis of eight eligible gene expression profiles of HCC led to 1,835 DEGs. Consistent with the fact that the cell cycle is closely related to various tumors, the functional annotation revealed that genes involved in the cell cycle were significantly enriched. A transcriptional regulatory network was constructed using the 62 TFs, which consisted of 872 TF-target interactions between 56 TFs and 672 DEGs in the context of HCC. The top 10 TFs covering the most downstream DEGs were ZNF354C, NFATC2, ARID3A, BRCA1, ZNF263, FOXD1, GATA3, FOXO3, FOXL1, and NR4A2. This network will appeal to future investigators focusing on the development of HCC. Conclusions: The transcriptional regulatory network can provide additional information that is valuable in understanding the underlying molecular mechanism in hepatic tumorigenesis. (Gut Liver 2017;11:112-120)
\end{abstract}

Key Words: Carcinoma, hepatocellular; Microarray dataset; Transcriptional regulatory network

\section{INTRODUCTION}

Hepatocellular carcinoma (HCC) is the most common and deadly form of liver cancer accounting for $\sim 80 \%$ of adult primary liver cancer, and it is one result of underlying liver disease. ${ }^{1}$ The prevalence and the incidence of HCC have progressively increased in the world recently. ${ }^{2}$ Because of the coexistence of cirrhosis and inflammation, early diagnosis of HCC is plagued by lack of clinical research and reliable indicators. Moreover, the lack of specific symptoms in the early stages of HCC also contributes to the poor prognosis of the disease.

Considering that, many researchers have pay attention to the HCC-specific biomarkers for early diagnosis of HCC currently, and a number of biomarkers have been identified. The most commonly used serological biomarker is $\alpha$-fetoprotein (AFP) for detection of HCC in clinical practice, which is a specific glycoprotein produced primarily by the fetal liver. ${ }^{3}$ However, due to its low sensitivity and specificity, the clinical diagnostic accuracy of AFP is unsatisfactory. It was then reported that combination assay of high-sensitivity des- $\gamma$-carboxy prothrombin and AFP-L3 can improve the detection rate of HCC. ${ }^{4}$ In addition, the midkine can serve as a useful marker in the diagnosis of AFP-negative HCCs and at a very early stage. ${ }^{5}$ Furthermore, several diagnostic biomarkers were identified continuously, such as Dickkopf- $1,{ }^{6}$ Golgi protein $73,{ }^{7}$ Glypican- $3,{ }^{8} \gamma$-glutamyl transferase, ${ }^{9} \alpha$-l-fucosidase, ${ }^{10}$ transforming growth factor $\beta-1,{ }^{11}$ IGFs, ${ }^{12}$ squamous cell carcinoma antigen ${ }^{13}$ osteopontin, ${ }^{14}$ heat shock proteins, ${ }^{15}$ and so on, among which most of them can be used together with AFP for diagnosis of HCC. Actually, combination of more than one biomarker may improve the accuracy of HCC diagnoses. ${ }^{16}$

Correspondence to: Zhenwen Qian

Department of Inspection, Affiliated Hospital of Jining Medical College of Shandong Province, No.79 Guhuai Road, Rencheng District, Jining 272029, China

Tel: +86-186-5371-9678, Fax: +86-0537-2903223, E-mail: qian_zhenwen@126.com

Received on February 2, 2016. Revised on February 19, 2016. Accepted on February 19, 2016. Published online July 27, 2016

pISSN 1976-2283 eISSN 2005-1212 https://doi.org/10.5009/gnl16063

Juan Chen and Zhenwen Qian contributed equally to this work as first authors.

(a) This is an Open Access article distributed under the terms of the Creative Commons Attribution Non-Commercial License (http://creativecommons.org/licenses/by-nc/4.0) which permits unrestricted non-commercial use, distribution, and reproduction in any medium, provided the original work is properly cited. 
Even so, none of the biomarkers have been considered as the reliable indicator in the early HCC diagnosis, which mainly because the pathogenesis of HCC remains undetermined. In this study, we extracted the gene expression profiles of HCC from Gene Expression Omnibus (GEO) database. By comparing the global gene expression profiles between HCC and the normal tissues, a set of differentially expressed genes (DEGs) were identified and the differentially expressed transcription factors (TFs) were further extracted. Additionally, the HCC-specific transcriptional regulatory network was constructed, which may provide better clues on the underlying regulatory mechanisms of pathogenesis of HCC and therapeutic applications.

\section{MATERIALS AND METHODS}

\section{Eligible datasets of HCC}

GEO database is a public functional genomics data repository (http://www.ncbi.nlm.nih.gov/geo/). ${ }^{17}$ By online search, the gene expression profiles of HCC were obtained from the GEO database. The following key words were used "carcinoma, hepatocellular" [MeSH Terms] OR hepatoma [All Fields] AND "Homo sapiens" [porgn] AND "Expression profiling by array" [Filter]. The eligible datasets were included and downloaded for integrated analysis, which were obtained from microarray experiments on the gene expression of HCC and normal tissues. In the eligible datasets, the etiologies for HCC patients were as follows: GSE17548 (10 HBV-related+3 HCV-related+2 cryptogenic HCC), GSE44074 (17 HBV-related+17 HCV-related), GSE45436 (93 cryptogenic HCC), GSE46408 (6 cryptogenic HCC), GSE50579 (8 HBV-related+9 HCV-related+14 alcohol-related+30 cryptogenic HCC), GSE57957 (39 cryptogenic HCC), GSE60502 (18 cryptogenic HCC), and GSE62232 (10 HBV-related+9 HCV-related+33 alcohol-related+29 cryptogenic HCC).

\section{Detection of DEGs}

All the raw data were preprocessed via background correction and normalization. The limma (linear models for microarray analysis) package in $\mathrm{R}$, one of the most commonly bioconduc- tor packages, was used to analyze the differential expression between HCC and the normal tissues by t-test. The p-value and false discovery rate (FDR) were also obtained. Genes with FDR $<0.01$ were defined as DEGs in this study.

\section{Functional annotation of DEGs}

To better understand the biological functions of DEGs in the pathogenesis of HCC, functional enrichment of DEGs were analyzed using the web-based tools in Database for Annotation, Visualization and Integrated Discovery (DAVID). ${ }^{18}$ The enriched gene ontology (GO) terms ${ }^{19}$ and Kyoto Encyclopedia of Genes and Genomes (KEGG) pathways ${ }^{20}$ were identified. Only the GO terms and KEGG pathways with $\mathrm{p}<0.05$ were taken into account as significantly enriched among the DEGs.

\section{Construction of transcriptional regulatory network}

TFs are essential for the regulation of gene expression, which can provide better clues on the underlying regulatory mechanisms. TRANSFAC (transcription factor database) is a manually curated database of eukaryotic TFs, their genomic binding sites and DNA binding profiles. Based on the DEGs in HCC from integrated analysis, we searched TRANSFAC for DEGs coded TFs and their targeted genes, and used TRANSFAC position weight matrix for gene promoter scanning ${ }^{21}$ to identify DEGs which have the binding sites of the TFs in the promoter regions. Then the transcriptional regulatory network was visualized using $\mathrm{Cy}$ toscape. ${ }^{22}$

\section{RNA preparation and qRT-PCR}

The peripheral blood samples were collected from five patients with HCC and five healthy volunteers, and the samples were immediately stored into vacuum EDTA anticoagulant tubes. All samples were obtained with permission, and the project was approved by our medical ethics committee for the relating screening, inspection, and data collection of the patients. Total RNA was extracted from the blood samples using the TRIzol ${ }^{\circledR}$ Reagent (Invitrogen, Carlsbad, CA, USA) following the manufacturer's protocols. Total RNA (1 $\mu \mathrm{g}$ ) was reverse-transcribed in $25 \mu \mathrm{L}$

Table 1. Characteristics of the Individual Studies

\begin{tabular}{|c|c|c|c|c|c|}
\hline GEO ID & Control & Case & Platform & Year & Author \\
\hline GSE17548 & 0 & 15 & GPL570 Affymetrix Human Genome U133 Plus 2.0 & 2009 & Yildiz et $a .^{35}$ \\
\hline GSE44074 & 0 & 34 & GPL13536 Kanazawa Univ. Human Liver chip 10k & 2013 & Ueda et ll $^{36}$ \\
\hline GSE45436 & 41 & 93 & GPL570 Affymetrix Human Genome U133 Plus 2.0 & 2013 & Wang et al. \\
\hline GSE46408 & 6 & 6 & GPL4133 Agilent-014850 Whole Human Genome Microarray 4×44K & 2013 & Jeng et al. \\
\hline GSE50579 & 7 & 61 & GPL14550 Agilent-028004 SurePrint G3 Human GE 8×60K Microarray & 2013 & Neumann et al. ${ }^{37}$ \\
\hline GSE57957 & 39 & 39 & GPL10558 Illumina HumanHT-12 V4.0 expression beadchip & 2014 & Mah et $a .^{38}$ \\
\hline GSE60502 & 18 & 18 & GPL96 Affymetrix Human Genome U133A Array & 2014 & Wang et al. $^{39}$ \\
\hline GSE62232 & 10 & 81 & GPL570 Affymetrix Human Genome U133 Plus 2.0 & 2014 & Zucman-Rossi et al. \\
\hline
\end{tabular}

GE0, Gene Expression Omnibus. 
Table 2. Top 15 Most Significantly Enriched Gene Ontology Terms of Differentially Expressed Genes

\begin{tabular}{|c|c|c|c|}
\hline GO ID & GO term & No. of genes & FDR \\
\hline \multicolumn{4}{|l|}{ Biological process } \\
\hline G0:0044281 & Small molecule metabolic process & 175 & $5.01 \mathrm{E}-18$ \\
\hline G0:0019752 & Carboxylic acid metabolic process & 107 & $3.42 \mathrm{E}-17$ \\
\hline G0:0043436 & Oxoacid metabolic process & 110 & $1.05 \mathrm{E}-15$ \\
\hline G0:0006082 & Organic acid metabolic process & 111 & $9.64 \mathrm{E}-16$ \\
\hline G0:0055114 & Oxidation-reduction process & 91 & $1.73 \mathrm{E}-14$ \\
\hline G0:0032787 & Monocarboxylic acid metabolic process & 66 & $5.40 \mathrm{E}-13$ \\
\hline G0:0009605 & Response to external stimulus & 78 & $9.74 \mathrm{E}-13$ \\
\hline G0:0044282 & Small molecule catabolic process & 47 & $5.50 \mathrm{E}-12$ \\
\hline G0:0016054 & Organic acid catabolic process & 43 & $4.91 \mathrm{E}-12$ \\
\hline G0:0046395 & Carboxylic acid catabolic process & 43 & $4.42 \mathrm{E}-12$ \\
\hline G0:0006629 & Lipid metabolic process & 96 & $3.63 \mathrm{E}-11$ \\
\hline G0:0050896 & Response to stimulus & 190 & $7.48 \mathrm{E}-10$ \\
\hline G0:0006631 & Fatty acid metabolic process & 44 & $1.35 \mathrm{E}-09$ \\
\hline G0:0006952 & Defense response & 66 & $3.47 \mathrm{E}-09$ \\
\hline G0:0033993 & Response to lipid & 55 & $6.00 \mathrm{E}-09$ \\
\hline \multicolumn{4}{|c|}{ Molecular function } \\
\hline G0:0004872 & Receptor activity & 67 & 7.67E-16 \\
\hline G0:0016491 & Oxidoreductase activity & 72 & $1.11 \mathrm{E}-13$ \\
\hline G0:0038023 & Signaling receptor activity & 54 & $2.31 \mathrm{E}-12$ \\
\hline G0:0004888 & Transmembrane signaling receptor activity & 48 & $2.58 \mathrm{E}-11$ \\
\hline G0:0060089 & Molecular transducer activity & 71 & $5.81 \mathrm{E}-11$ \\
\hline G0:0004871 & Signal transducer activity & 57 & $1.27 \mathrm{E}-07$ \\
\hline G0:0005102 & Receptor binding & 62 & $2.30 \mathrm{E}-05$ \\
\hline G0:0005125 & Cytokine activity & 16 & $2.91 \mathrm{E}-05$ \\
\hline G0:0004930 & G-protein coupled receptor activity & 22 & $1.09 \mathrm{E}-04$ \\
\hline G0:0048037 & Cofactor binding & 39 & $2.21 \mathrm{E}-04$ \\
\hline G0:0004252 & Serine-type endopeptidase activity & 11 & $2.23 \mathrm{E}-04$ \\
\hline G0:0008236 & Serine-type peptidase activity & 12 & $6.36 \mathrm{E}-04$ \\
\hline G0:0017171 & Serine hydrolase activity & 12 & 5.87E-04 \\
\hline G0:0004497 & Monooxygenase activity & 11 & $6.44 \mathrm{E}-04$ \\
\hline G0:0043177 & Organic acid binding & 21 & $9.70 \mathrm{E}-04$ \\
\hline \multicolumn{4}{|c|}{ Cellular component } \\
\hline G0:0005576 & Extracellular region & 70 & $1.36 \mathrm{E}-14$ \\
\hline G0:0016021 & Integral component of membrane & 198 & $7.85 \mathrm{E}-15$ \\
\hline G0:0031224 & Intrinsic component of membrane & 166 & $5.96 \mathrm{E}-15$ \\
\hline G0:0044425 & Membrane part & 262 & $7.70 \mathrm{E}-15$ \\
\hline G0:0044459 & Plasma membrane part & 102 & $3.74 \mathrm{E}-13$ \\
\hline G0:0031226 & Intrinsic component of plasma membrane & 67 & $7.56 \mathrm{E}-13$ \\
\hline G0:0044421 & Extracellular region part & 205 & $6.79 \mathrm{E}-13$ \\
\hline G0:0005887 & Integral component of plasma membrane & 65 & $3.95 \mathrm{E}-12$ \\
\hline G0:0005615 & Extracellular space & 72 & $7.80 \mathrm{E}-12$ \\
\hline G0:0043230 & Extracellular organelle & 153 & $1.78 \mathrm{E}-09$ \\
\hline G0:1903561 & Extracellular vesicle & 153 & $1.62 \mathrm{E}-09$ \\
\hline G0:0065010 & Extracellular membrane-bounded organelle & 152 & $2.50 \mathrm{E}-09$ \\
\hline G0:0070062 & Extracellular exosome & 152 & $2.31 \mathrm{E}-09$ \\
\hline G0:0031988 & Membrane-bounded vesicle & 165 & $1.54 \mathrm{E}-08$ \\
\hline G0:0005886 & Plasma membrane & 127 & $1.74 \mathrm{E}-08$ \\
\hline
\end{tabular}

Go, gene ontology; FDR, false discovery rate. 
reactions using SuperScript ${ }^{\circledR}$ III Reverse Transcriptase (Invitrogen), and the resulted cDNA was used as template for real-time polymerase chain reaction (PCR). Real-time PCR was carried out in ABI 7500 real-time PCR system with a Power SYBR ${ }^{\circledR}$ Green PCR Master Mix (Invitrogen). The results were analyzed using $2^{-\Delta \Delta c t}$ method. ACTIN gene was used as the endogenous control. The sequences of primers used for real-time PCR were listed in Supplementary Table 1.

\section{RESULTS}

\section{Comparison of global gene expression profiles of HCC and normal tissue}

In present study, eight eligible gene expression profiles of HCC were included, and the detailed information of datasets was displayed in Table 1. Totally, 347 cases of HCCs (45 HBVrelated+38 HCV-related+47 alcohol-related+217 cryptogenic HCC) and 121 controls of normal liver tissues were enrolled in the integrated analysis. A set of 1,835 genes were regarded as having DEG by applying the selection criteria of FDR $<0.01$, among which 1,145 were upregulated and 690 downregulated,
Table 3. Top 15 Most Significantly Enriched Kyoto Encyclopedia of Genes and Genomes Pathways of Differentially Expressed Genes

\begin{tabular}{lcc}
\hline \multicolumn{1}{c}{ KEGG term } & Count & FDR \\
\hline Cell cycle & 35 & $1.06 \mathrm{E}-14$ \\
Valine, leucine and isoleucine degradation & 20 & $1.29 \mathrm{E}-12$ \\
Fatty acid metabolism & 16 & $7.88 \mathrm{E}-09$ \\
Propanoate metabolism & 14 & $1.22 \mathrm{E}-08$ \\
DNA replication & 14 & $4.34 \mathrm{E}-08$ \\
Oocyte meiosis & 24 & $6.58 \mathrm{E}-08$ \\
p53 signaling pathway & 18 & $1.86 \mathrm{E}-07$ \\
Progesterone-mediated oocyte maturation & 19 & $1.35 \mathrm{E}-06$ \\
Pathways in cancer & 41 & $1.88 \mathrm{E}-06$ \\
Lysine degradation & 14 & $2.30 \mathrm{E}-06$ \\
Mineral absorption & 14 & $3.40 \mathrm{E}-06$ \\
Pyrimidine metabolism & 19 & $3.63 \mathrm{E}-06$ \\
Homologous recombination & 10 & $5.36 \mathrm{E}-06$ \\
Tryptophan metabolism & 12 & $1.03 \mathrm{E}-05$ \\
Pyruvate metabolism & 11 & $4.26 \mathrm{E}-05$ \\
\hline
\end{tabular}

KEGG, Kyoto Encyclopedia of Genes and Genomes; FDR, false discovery rate.

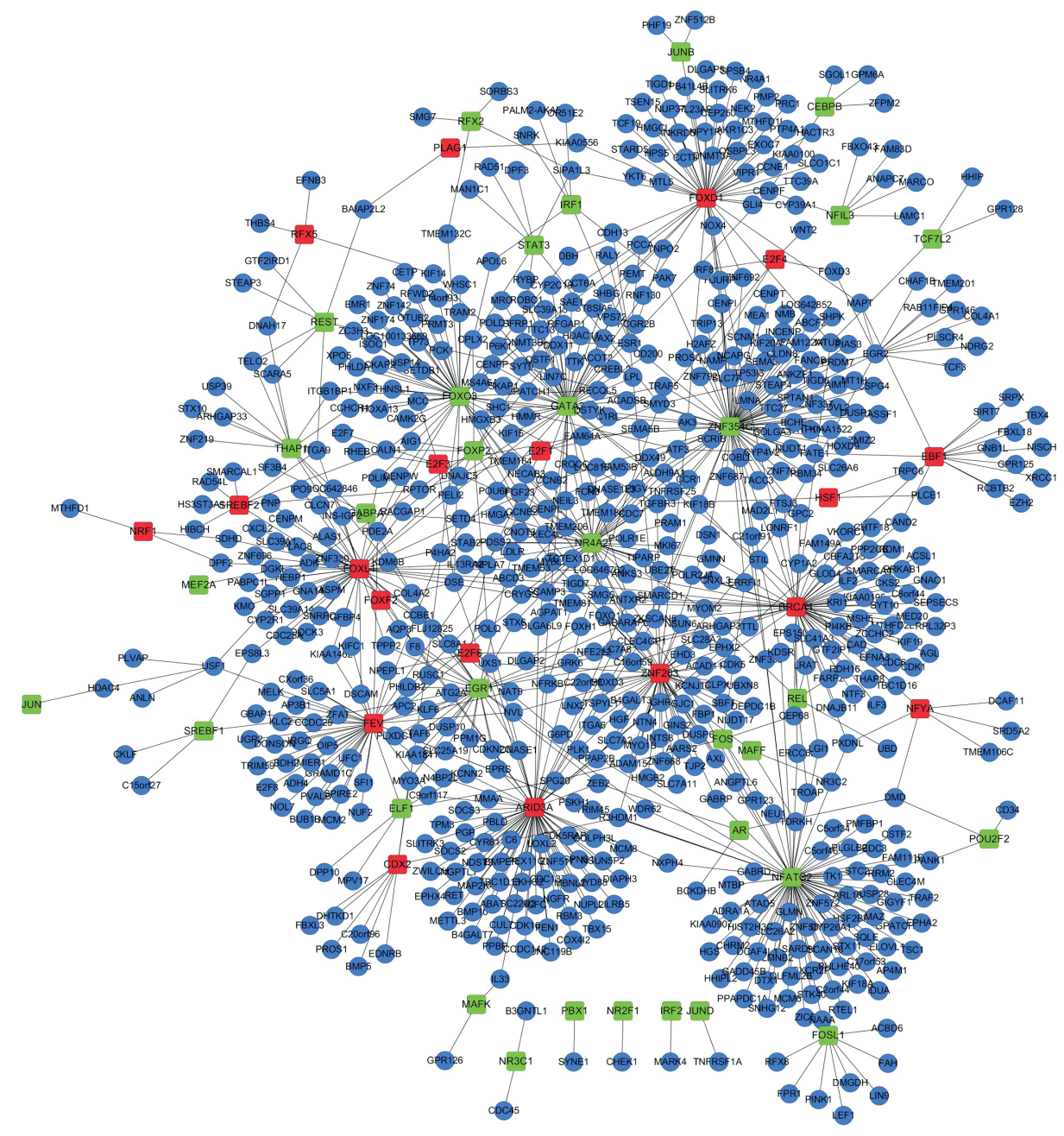

Fig. 1. The established transcriptional regulatory network of hepatocellular carcinoma. Red and green nodes denote upregulated and downregulated transcription factors (TFs), respectively. Blue nodes denote differentially expressed genes predicted to interact with the corresponding TFs. 
respectively. All the DEGs were listed in Supplementary Table 2.

\section{Functional enrichment of DEGs}

By subjecting the DEGs to enrichment analysis on gene sets based on GO (biological process, cellular component, and molecular function) and KEGG pathways, we observed that DEGs were significantly enriched in various terms (Table 2). For biological process, the DEGs were mainly enriched in oxidationreduction process, response to external stimulus, response to stimulus and defense response. For cellular component, extracellular region and integral component of membrane were involved. For molecular function, receptor activity and oxidoreductase activity were implicated. Based on the canonical signaling pathways documented in KEGG, pathways on p53 signaling pathway, pathways in cancer, cell cycle, DNA replication and homologous recombination were significantly enriched (Table 3).

\section{Transcriptional regulatory network}

Transcriptional regulatory network is a directed graph describing regulatory effect of TFs on the expression of target genes. Based on the database of TRANSFAC, 62 differentially expressed TFs were identified, in which 23 were upregulated and 39 were downregulated. The constructed regulatory network consisted of 872 TF-target interactions between 56 TFs and 672 DEGs in the context of HCC (Fig. 1). The top 10 TFs covering the most downstream DEGs were identified as crucial TFs involved in the development of HCC and listed in Table 4, including ZNF354C, NFATC2, ARID3A, BRCA1, ZNF263, FOXD1, GATA3, F0X03, FOXL1, and NR4A2.

Table 4. Top 10 Transcription Factors Covering the Most Downstream Differentially Expressed Genes Involved in the Development of Hepatocellular Carcinoma

\begin{tabular}{|c|c|c|c|c|}
\hline $\begin{array}{l}\text { Transcription } \\
\text { factor }\end{array}$ & $\log \mathrm{FC}$ & $\begin{array}{l}\text { Up/ } \\
\text { down }\end{array}$ & Count & Genes \\
\hline \multirow[t]{8}{*}{ ZNF354C } & $-2.12 \mathrm{E}+00$ & Down & 81 & TMEM206,TNFRSF25,STEAP4,FANCB,FATE1,NOX4,RECQL5,H2AFZ,ZNF687,FTSJ3,ZN \\
\hline & & & & F335,SYT9,PAK7,AIM1L,PROSC,CCNE2,SHPK,TCTEX1D1,DVL2,CCNB2,ESR1,ZNF337, \\
\hline & & & & SEMA5B,TRAF5,ZKSCAN5,ERRFI1,ZMIZ2,CSPG4,ANKZF1,MAD2L1,SMARCD1,NUDT \\
\hline & & & & 1,CYP4V2,ATP11C,PRDM7,ALDH9A1,NMB,GPC2,HJURP,SLC7A6,DSTYK,KIAA1522,D \\
\hline & & & & DX49,GOLGA3,TP53I3,EGR2,TACC3,TRIP13,TTC27,FAM64A,ZNF76,SEMA3F,KDSR,SL \\
\hline & & & & C26A6,SPTAN1,SCNM1,DSN1,PIAS3,DUSP1,SCRIB,ABCF2,FAM122A,CCR1,NAMPT,Z \\
\hline & & & & NF692,LOC642852,KIF20A,RASSF1,HOXD9,NCAPG,CENPI,BCHE,CLDN8,RBM34,MT1 \\
\hline & & & & H,COBLL1,INCENP,CENPT,MEA1,C1RL,TIGD6 \\
\hline \multirow[t]{8}{*}{ NFATC2 } & $-2.21 \mathrm{E}+00$ & Down & 78 & SNHG12,EPHA2,KIF18A,DTX1,BHLHE40,C21orf91,KDSR,WDR62,NXPH4,LGI1,CSTF2, \\
\hline & & & & C17orf53,STC2,DUSP28,SARDH,GABRD,PXDNL,HIST2H3C,ELOVL1,GLMN,PSKH1,TD \\
\hline & & & & RKH,KIAA0907,GIGYF1,GADD45B,ANGPTL6,SQLE,PPAPDC1A,ZNF581,SLC26A2,C5o \\
\hline & & & & rf46,G6PD,MAZ,MCM6,TRAF2,LMNB2,CHRM2,TROAP,CLEC4GP1,ZIC5,NAAA,HGS,A \\
\hline & & & & TAD5,R3HDM1,STK40,GABRP,PANK1,PMFBP1,CLEC4M,ADRA1A,MTBP,STX11,ZSCA \\
\hline & & & & N16,ZNF572,CYP26A1,C5orf34,ARL16,PLGLB2,HHIPL2,NEU1,FAM111B,ERCC6L,GPA \\
\hline & & & & TCH4,TK1,TSC1,RRM2,AP4M1,OLFML2B,TRIM45,CXCR2P1,RTEL1,C2orf44,DCAF4L1, \\
\hline & & & & HSF2BP,FBP1,ZNF335,EDC3,IDUA \\
\hline \multirow[t]{7}{*}{ ARID3A } & $1.60 \mathrm{E}+00$ & Up & 74 & ANTXR2,PLEKHG2,NXPH4,EPRS,MYD88,B4GALT7,RBM3,EPHX4,PPBP,MBNL2,CDK1 \\
\hline & & & & 6,DSE,ZNF517,N4BP2L1,DIAPH3,PLK1,HCFC1,METTL3,AGPAT1,TTL,NUPL2,ATG2A,S \\
\hline & & & & MARCD1,ANGPTL1,NGFR,KCNN2,MCM8,RET,TPM3,LILRB5,TBC1D13,MMAA,F0XH1, \\
\hline & & & & ZKSCAN5,DNASE1,F8,BMPER,SOCS3,UXS1,IL33,CCDC137,SLC7A8,SOCS2,PGP,NSUN \\
\hline & & & & 5P2,ZWILCH,BMP10,GOLPH3L,MAP2K4,GHR,PEX11G,POLQ,TSC22D2,CDKN2C,CDK5 \\
\hline & & & & RAP1,L0XL2,GOLGA6L9,ZEB2,CYR61,PPAP2B,FEN1,C9orf117,UNC119B,C6,NDST3,P \\
\hline & & & & BLD,CUL7,COX4I2,SPNS1,CCDC142,SLITRK3,ABAT,TBX15,NFRKB \\
\hline \multirow[t]{6}{*}{ BRCA1 } & $9.55 \mathrm{E}-01$ & Up & 64 & LOC646762,ANTXR2,POLR2J4,CCDC64,GNA01,PI4KB,CBFA2T3,TIGD7,GPC2,MAD2L1, \\
\hline & & & & KRI1,RDH16,GMNN,CEP68,TBC1D16,CYP1A2,PCNXL3,CDK1,SMG5,KIAA0195,C8orf4 \\
\hline & & & & 4,NTF3,PXDNL,NFE2L2,DNAJB11,LMNA,C16orf59,CKS2,KIF19,EFNA3,ILF2,SEPSECS, \\
\hline & & & & TMEM81,FTSJ3,ERCC6L,MED20,SMARCA4,GLOD4,GABARAPL1,MTUS2,GHR,CAND2, \\
\hline & & & & ACSL1,PRKAB1,ZCCHC2,KIF18B,GTF2IP1,FARP2,AGL,LGI1,VKORC1,THAP8,PPP2CB, \\
\hline & & & & MTHFD2L,ILF3,CHTF18,RPL32P3,FAM149A,CAD,MSH5,GRK6,LRAT,SYT10,RDM1 \\
\hline
\end{tabular}


Table 4. Continued

\begin{tabular}{|c|c|c|c|c|}
\hline $\begin{array}{l}\text { Transcription } \\
\text { factor }\end{array}$ & $\log F C$ & $\begin{array}{l}\text { Up/ } \\
\text { down }\end{array}$ & Count & Genes \\
\hline FOXD1 & $2.46 \mathrm{E}+00$ & Up & 52 & $\begin{array}{l}\text { PRC1,NPY1R,NR4A1,NUP37,NEK2,HMGCL,SPSB4,TTC39A,CCNE1,RPL23AP7,PMP2,C } \\
\text { YP39A1,VIPR1,MAPT,AKR1C3,DBH,GLI4,YKT6,MTHFD1L,SLC01C1,SIPA1L3,ZNF692, } \\
\text { EX0C7,LMNA,C21orf91,TCF19,STARD5,SLITRK6,PEMT,CEP250,EPB41L4B,DNMT3A,C } \\
\text { CT6A,HPS5,ST8SIA6,CCT3,OSBPL3,TIGD1,RNF130,PHACTR3,MTL5,TSEN15,CENPF,M } \\
\text { TUS2,KIAA0556,HJURP,ANKRD55,AK3,KIAA0100,PTP4A1,SAE1,DLGAP5 }\end{array}$ \\
\hline ZNF263 & $3.40 \mathrm{E}-01$ & Up & 52 & $\begin{array}{l}\text { H0XD3,EPHX2,SBF1,NVL,DUSP6,SLC7A11,AARS2,R3HDM1,CLEC4G,NEIL3,UBXN8,L } \\
\text { NX2,MYBL2,ARHGAP39,ITGA6,TJP2,KCNJ16,B4GALT3,NTN4,EHD3,PLK1,ADAM15,A } \\
\text { XL,TSPYL1,NAT9,GJC1,INTS8,DSN1,MY01B,CDK5,SLC7A2,CLPX,GABRP,TGFBR3,HM } \\
\text { GB2,C22orf23,PPAP2B,F0X01,HGF,NFE2L2,LONRF1,GINS2,ESR1,SLC28A3,NSUN6,U } \\
\text { XS1,ANGPTL6,ACAD11,ZNF668,DEPDC1B,AK3,NUDT17 }\end{array}$ \\
\hline F0X03 & $-3.95 \mathrm{E}-01$ & Down & 51 & $\begin{array}{l}\text { CALN1,XP05,CKAP2,CLEC4G,DNMT3B,TMEM132C,LOC100133669,CCHCR1,PDLIM5, } \\
\text { C14orf93,ZNF74,CAMK2G,RECQL5,DSTYK,C1RL,PRMT3,THNSL1,CPLX2,MCC,DSE,ITG } \\
\text { B1BP1,DBH,PHLDA1,HOXA13,ZC3H3,ISOC1,ATF3,FLJ12825,CENPP,ZNF174,AP0L6,Z } \\
\text { NF142,TMEM164,RFWD2,CCNE2,PCK1,CENPW,CLEC4GP1,TP73,DUSP14,NXF3,SAE1, } \\
\text { TRAM2,NEIL3,OTUB2,CETP,PDE2A,TGFBR3,EMR1,WHSC1,SETDB1 }\end{array}$ \\
\hline GATA3 & $-1.75 \mathrm{E}+00$ & Down & 51 & $\begin{array}{l}\text { SHC1,LPL,ANTXR2,HMGXB3,FCGR2B,TMEM206,SAE1,CENPP,CREBL2,LDLR,KIF18B, } \\
\text { MR0,DDX11,PELI2,SHBG,RNF130,CD200,P4HA2,NEIL3,CENPF,DNAJC5,GOLGA6L9,A } \\
\text { CADSB,SLC39A13,HDAC11,C1RL,PEMT,TRAF5,OSTF1,TTC13,R0B01,SKAP1,IP6K1,H } \\
\text { MMR,KIF15,MS4A6A,SLC7A8,GPATCH1,SFRP1,VPS72,ARFGAP1,STIL,ACOT2,CYP2C } \\
\text { 19,KIF14,TTK,RYBP,VAX2,AGPAT1,P0LD1,LIN7C }\end{array}$ \\
\hline F0XL1 & $1.37 \mathrm{E}+00$ & Up & 51 & $\begin{array}{l}\text { CLCN7,PDLIM5,INS-IGF2,IL13RA2,DNAJC5,IP09,DSCAM,SGPP1,PNP,TIGD7,TMEM81, } \\
\text { AQP3,DPF2,ZNF330,GNA14,KIFC1,SNRPC,LOC646762,PLAC8,ASPM,SLC39A14,RACG } \\
\text { AP1,CCBE1,STAB2,SMG5,LOC642846,IGFBP4,PELI2,G6PD,ITGA9,TPPP2,DGKI,ATG2A, } \\
\text { CENPW,HEBP1,CENPM,PABPC1L,SDHD,CYP2R1,ALAS1,KM0,EPS8L3,KIAA1462,CCN } \\
\text { B2,ZNF696,ADK,CXCL2,COL4A2,SLC39A1,CDC25A,D0CK3 }\end{array}$ \\
\hline NR4A2 & $-2.29 \mathrm{E}+00$ & Down & 43 & $\begin{array}{l}\text { CENPL,FBP1,DDX49,F0X01,UBE2T,ABCD3,ALDH9A1,CROCC,CCR1,FGF23,IL13RA2,A } \\
\text { NKS3,SETD4,FAM53B,PRAM1,PDSS2,NFRKB,TMEM184B,POU6F2,TMEM164,FCN3,M } \\
\text { KI67,STX6,SCAMP3,STAB2,HMGA1,MYOM2,TNFRSF25,TIPARP,LOC81691,POLR2J4, } \\
\text { CDC7,CRYGS,ATF3,GABARAPL1,NECAB3,DNASE1L3,CNOT3,GMNN,TMEM53,PIGV,P } \\
\text { CNXL3,POLR1E }\end{array}$ \\
\hline
\end{tabular}

\section{Validation of TF-target by qRT-PCR}

To validate the findings in the integrated analysis, the peripheral blood samples were used and a pair of TF-target was selected, including ZNF263 and NEIL3. The results of qRT-PCR showed that the expression pattern of selected genes in HCC were similar with that in the integrated analysis (Fig. 2). ZNF263 and NEIL3 were both upregulated in the blood of HCC patient compared with the healthy volunteers.

\section{DISCUSSION}

HCC is a complex disease that involves various molecule interactions. Chronic infection of HBV or HCV is a major risk factor in the development of the HCC, independently from alco- hol abuse and metabolic disease. Moreover, the HBV and HCV infection can cause the disarrangement in cellular pathways through an indirect and/or direct mechanism in liver injury. This study was designed to determine their common mechanism by integrating sufficient number of the HCC samples with various etiologies. ${ }^{23}$

In the present study, integrated analysis of eight microarray data of HCC led to a set of 1,835 DEGs (1,145 upregulated and 690 downregulated) in HCC compared with normal tissues. Functional annotation showed that DEGs were closely related to common pathways for cancers, including p53 signaling pathway and pathways in cancer. Previous study suggested that viral hepatitis infection was associated with cellular inflammation, oxidative stress, and DNA damage, that may lead to subsequent hepatic injuries such as chronic hepatitis, fibrosis, cirrhosis, and 

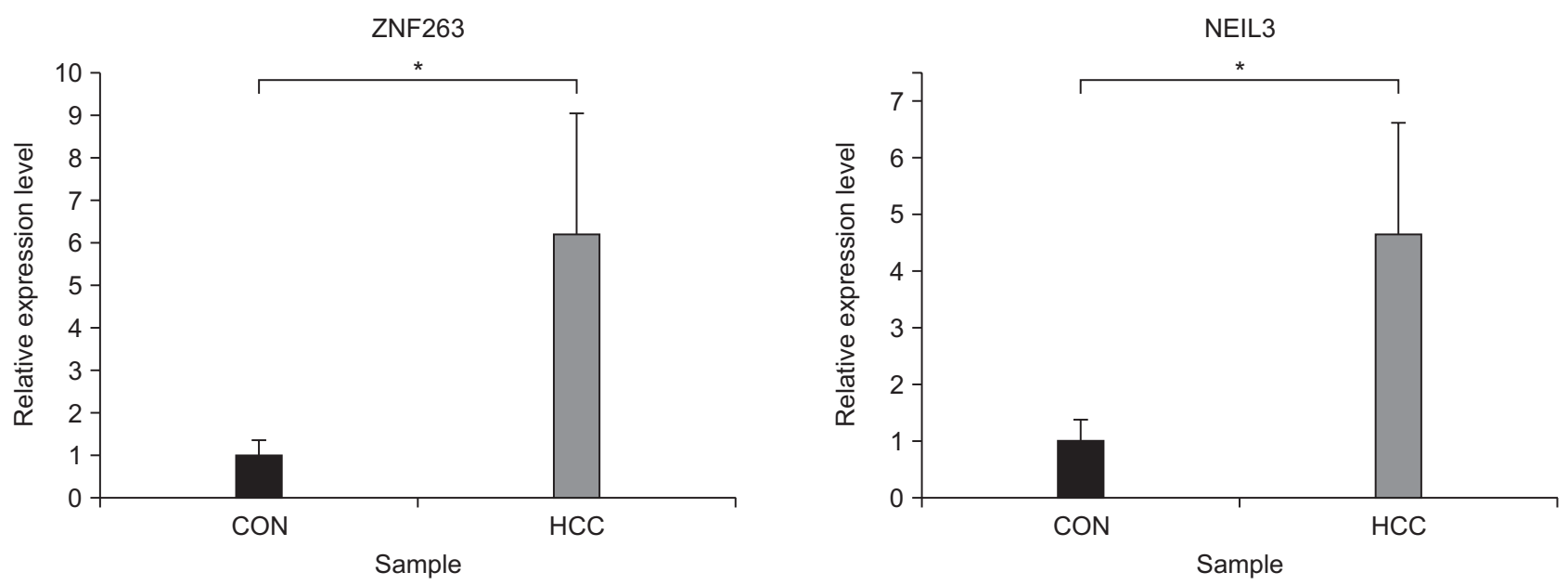

Fig. 2. qRT-PCR validation of a transcription factor-target pair in peripheral blood collected from five patients with hepatocellular carcinoma (HCC) and five healthy volunteers. Actin was used as an internal reference gene for normalization. CON, control (normal tissues). * $\mathrm{p}<0.05$.

finally $\mathrm{HCC}^{23}$ Our results revealed that cell cycle, DNA replication and homologous recombination were significantly enriched, which may be due to the DNA damage. Moreover, DEGs were significantly enriched in various GO terms, such as oxidationreduction process, response to external stimulus, response to stimulus, defense response, and oxidoreductase activity, which may be due to the cellular inflammation and oxidative stress in the process of viral hepatitis infection.

Network analysis allows structured grouping of genes, and network construction is an important stage in the pathogenesis studies. A study of core network in HCC revealed that miRNAs mainly regulate biological functions related to mitochondria and oxidative reduction, while TFs mainly regulate immune responses, extracellular activity and the cell cycle. ${ }^{24} \mathrm{~A}$ recent study suggested that 86 crosstalks involving 52 pathways were identified through the DEGs between adjacent nontumor and HCC samples. ${ }^{25}$ Totally, 62 differentially expressed TFs were identified in this study, and the transcriptional regulatory network was constructed, which consisted of 872 TF-target interactions between 56 TFs and 672 DEGs in the context of HCC. The HCC-specific transcriptional regulatory network may help to better understand its underlying molecular mechanism of pathogenesis and tumorigenesis of HCC.

Based on the constructed transcriptional regulatory network, a set of crucial TFs caught our attention, which covered the most downstream DEGs, including ZNF354C, NFATC2, ARID3A, BRCA1, ZNF263, FOXD1, GATA3, FOX03, FOXL1, and NR4A2. Previous studies reported that the forkhead box 0 (FOXO) TFs are involved in various cancer development including HCC, suggesting that FOXO factors function as tumor suppressors in a variety of cancers. ${ }^{26,27}$ As an important member of FOXO family, threonine 32 (Thr32) of FOXO3 is critical for TGF- $\beta$ induced apoptosis via Bim in $\mathrm{HCC}^{28}$ Our results showed that F0X03 was down-regulated in HCC compared with the normal tissues, which provided additional evidence for FOX03 playing a role in HCC. Up to now, little was known about the function of ZNF263. The results of a recent study revealed that ZNF263 may be closely related to the stress- and age-related diseases, and it can have both positive and negative effects on transcriptional regulation of its target genes. ${ }^{29}$ Herein, we found that ZNF263 was one of the significantly upregulated TFs, indicating that it may appeal to future investigators to study this TF in the development of the many complex diseases including HCC.

To extract more information about the crucial TFs involved in HCC, the functions of their targets were further explored. NEIL3 (nei endonuclease VIII-like 3) is a kind of DNA glycosylase, which can initiate base excision repair by hydrolysing the $\mathrm{N}$ glycosidic bond and releasing the damaged base. ${ }^{30}$ It was reported that the genetic abnormalities of NEIL3 may be related to hepatocarcinogenesis. ${ }^{31}$ We found that NEIL3 was upregulated in HCC compared with normal tissues. CLEC4G is a member of Ctype lectins, which are important in various immune functions, including inflammation and immunity to tumor and virally infected cells. CLEC4G was predominantly expressed in liver and was expressed at very low levels or even undetectable in liver cancer tissue. ${ }^{32}$ CLEC4G was a top-listing downregulated gene, which suggested that disruption of expression of these C-type lectin proteins may have a role in the pathogenesis of HCC. ${ }^{33}$ HCC is a highly vascularized tumor and it can be amenable to antiangiogenic treatment modalities. The vascular remodelling and endothelial transdifferentiation are major pathogenic events in HCC development, in which Stabilin-2 may play the important role. ${ }^{34}$

The present study provided useful information on the transcriptomic landscape and to the constructed transcriptional regulatory network will be hopeful to better understand its underlying molecular mechanism in hepatic tumorigenesis. These findings shed light on several important TFs targets that may 
potentially drive hepatocarcinogenesis, and further functional characterization are needed to verify our findings.

\section{CONFLICTS OF INTEREST}

No potential conflict of interest relevant to this article was reported.

\section{REFERENCES}

1. Villanueva A, Llovet JM. Liver cancer in 2013. Mutational landscape of HCC: the end of the beginning. Nat Rev Clin Oncol 2014; 11:73-74.

2. El-Serag HB. Epidemiology of viral hepatitis and hepatocellular carcinoma. Gastroenterology 2012;142:1264-1273.e1.

3. Stefaniuk P, Cianciara J, Wiercinska-Drapalo A. Present and future possibilities for early diagnosis of hepatocellular carcinoma. World J Gastroenterol 2010;16:418-424.

4. Sassa T, Kumada T, Nakano S, Uematsu T. Clinical utility of simultaneous measurement of serum high-sensitivity des-gammacarboxy prothrombin and Lens culinaris agglutinin A-reactive alpha-fetoprotein in patients with small hepatocellular carcinoma. Eur J Gastroenterol Hepatol 1999;11:1387-1392.

5. Zhu WW, Guo JJ, Guo L, et al. Evaluation of midkine as a diagnostic serum biomarker in hepatocellular carcinoma. Clin Cancer Res 2013;19:3944-3954.

6. Prieto PA, Cha CH. DKK1 as a serum biomarker for hepatocellular carcinoma. Hepatobiliary Surg Nutr 2013;2:127-128.

7. Mao Y, Yang $\mathrm{H}, \mathrm{Xu} \mathrm{H}$, et al. Golgi protein 73 (GOLPH2) is a valuable serum marker for hepatocellular carcinoma. Gut 2010;59:1687-1693.

8. Badr EA, Korah TE, Ghani AA, El-Sayed S, Badr S. Role of serum glypican-3 in the diagnosis and differentiation of small hepatocellular carcinoma from hepatitis-C virus cirrhosis. Alexandria J Med 2014;50:221-226.

9. Stefaniuk P, Mikuła T, Krygier R, Dusza M, Cianciara J, Wiercińska-Drapało A. Combination of alpha-fetoprotein with gamma-glutamyl transferase as the complementary biomarkers useful in early diagnosis of hepatocellular carcinoma. Exp Clin Hep 2010;6:40-44.

10. Gan Y, Liang Q, Song X. Diagnostic value of alpha-L-fucosidase for hepatocellular carcinoma: a meta-analysis. Tumour Biol 2014; 35:3953-5960.

11. Yasmin Anum MY, Looi ML, Nor Aini AH, et al. Combined assessment of TGF-beta-1 and alpha-fetoprotein values improves specificity in the diagnosis of hepatocellular carcinoma and other chronic liver diseases in Malaysia. Med J Malaysia 2009;64:223227.

12. Lopez JB. Recent developments in the first detection of hepatocellular carcinoma. Clin Biochem Rev 2005;26:65-79.

13. Giannelli G, Marinosci F, Trerotoli P, et al. SCCA antigen combined with alpha-fetoprotein as serologic markers of HCC. Int J
Cancer 2005;117:506-509.

14. Hafez SS, El Sebai AA, Abd AL Aziz MM, et al. Alfa-fetoprotein L3 subfraction and osteopontin: novel markers for the diagnosis of hepatocellular carcinoma. J Am Sci 2013;9:322-328.

15. Gruden G, Carucci P, Lolli V, et al. Serum heat shock protein 27 levels in patients with hepatocellular carcinoma. Cell Stress Chaperones 2013;18:235-241.

16. Khattab M, Fouad M, Ahmed E. Role of biomarkers in the prediction and diagnosis of hepatocellular carcinoma. World J Hepatol 2015;7:2474-2481.

17. Barrett T, Wilhite SE, Ledoux P, et al. NCBI GEO: archive for functional genomics data sets: update. Nucleic Acids Res 2013;41(Database issue):D991-D995.

18. Huang DW, Sherman BT, Tan Q, et al. The DAVID Gene Functional Classification Tool: a novel biological module-centric algorithm to functionally analyze large gene lists. Genome Biol 2007;8:R183.

19. Young MD, Wakefield MJ, Smyth GK, Oshlack A. Gene ontology analysis for RNA-seq: accounting for selection bias. Genome Biol 2010;11:R14.

20. Kanehisa M, Goto S. KEGG: Kyoto encyclopedia of genes and genomes. Nucleic Acids Res 2000;28:27-30.

21. Knüppel R, Dietze P, Lehnberg W, Frech K, Wingender E. TRANSFAC retrieval program: a network model database of eukaryotic transcription regulating sequences and proteins. J Comput Biol 1994;1:191-198.

22. Shannon P, Markiel A, Ozier 0, et al. Cytoscape: a software environment for integrated models of biomolecular interaction networks. Genome Res 2003;13:2498-2504.

23. Sukowati CH, El-Khobar KE, Ie SI, Anfuso B, Muljono DH, Tiribelli C. Significance of hepatitis virus infection in the oncogenic initiation of hepatocellular carcinoma. World J Gastroenterol 2016;22:1497-1512.

24. Gu Z, Zhang C, Wang J. Gene regulation is governed by a core network in hepatocellular carcinoma. BMC Syst Biol 2012;6:32.

25. Liang J, Lv J, Liu Z. Identification of dysfunctional biological pathways and their synergistic mechanism in hepatocellular carcinoma process. Exp Mol Pathol 2015;98:540-545.

26. Fu Z, Tindall DJ. FOXOs, cancer and regulation of apoptosis. Oncogene 2008;27:2312-2319.

27. Fei M, Lu M, Wang Y, et al. Arsenic trioxide-induced growth arrest of human hepatocellular carcinoma cells involving F0X03a expression and localization. Med Oncol 2009;26:178-185.

28. Zhao X, Liu Y, Du L, et al. Threonine 32 (Thr32) of Fox03 is critical for TGF- $\beta$-induced apoptosis via Bim in hepatocarcinoma cells. Protein Cell 2015;6:127-138.

29. Frietze S, Lan X, Jin VX, Farnham PJ. Genomic targets of the $\mathrm{KRAB}$ and SCAN domain-containing zinc finger protein 263. J Biol Chem 2010;285:1393-1403.

30. Takao M, Oohata Y, Kitadokoro K, et al. Human Nei-like protein NEIL3 has AP lyase activity specific for single-stranded DNA and confers oxidative stress resistance in Escherichia coli mutant. Genes Cells 2009;14:261-270. 
31. Zhang H, Ma H, Wang Q, et al. Analysis of loss of heterozygosity on chromosome $4 \mathrm{q}$ in hepatocellular carcinoma using highthroughput SNP array. Oncol Rep 2010;23:445-455.

32. Uhlen M, Oksvold P, Fagerberg L, et al. Towards a knowledgebased Human Protein Atlas. Nat Biotechnol 2010;28:1248-1250.

33. Ho DW, Kai AK, Ng IO. TCGA whole-transcriptome sequencing data reveals significantly dysregulated genes and signaling pathways in hepatocellular carcinoma. Front Med 2015;9:322-330.

34. Nonaka H, Sugano S, Miyajima A. Serial analysis of gene expression in sinusoidal endothelial cells from normal and injured mouse liver. Biochem Biophys Res Commun 2004;324:15-24.

35. Yildiz G, Arslan-Ergul A, Bagislar S, et al. Genome-wide transcriptional reorganization associated with senescence-to-immortality switch during human hepatocellular carcinogenesis. PLoS One 2013;8:e64016.

36. Ueda T, Honda M, Horimoto K, et al. Gene expression profiling of hepatitis B- and hepatitis C-related hepatocellular carcinoma using graphical Gaussian modeling. Genomics 2013;101:238-248.

37. Neumann 0, Kesselmeier M, Geffers R, et al. Methylome analysis and integrative profiling of human HCCs identify novel protumorigenic factors. Hepatology 2012;56:1817-1827.

38. Mah WC, Thurnherr T, Chow PK, et al. Methylation profiles reveal distinct subgroup of hepatocellular carcinoma patients with poor prognosis. PLoS One 2014;9:e104158.

39. Wang YH, Cheng TY, Chen TY, Chang KM, Chuang VP, Kao KJ. Plasmalemmal Vesicle Associated Protein (PLVAP) as a therapeutic target for treatment of hepatocellular carcinoma. BMC Cancer 2014;14:815. 